\title{
Efficient Synthesis of Single Chain Globules Mimicking the Morphology and Polymerase Activity of Metalloenzymes ${ }^{a}$
}

Ana Sanchez-Sanchez, Arantxa Arbe, Joachim Kohlbrecher, Juan Colmenero, José A. Pomposo*

Dr. A. Sanchez-Sanchez

Centro de Física de Materiales (CSIC, UPV/EHU) - Materials Physics Center, Paseo Manuel de Lardizábal 5, 20018 San Sebastián, Spain, Departamento de Física de Materiales, Universidad del País Vasco (UPV/EHU), Apartado 1072, 20800 San Sebastián, Spain

Prof. A. Arbe

Centro de Física de Materiales (CSIC, UPV/EHU) - Materials Physics Center, Paseo Manuel de Lardizábal 5, 20018 San Sebastián, Spain

Dr. J. Kohlbrecher

Laboratory for Neutron Scattering, Paul Scherrer Institut, CH-5232 Villigen, Switzerland.

Prof. J. Colmenero

Centro de Física de Materiales (CSIC, UPV/EHU) - Materials Physics Center, Paseo Manuel de Lardizabal 5, 20018 San Sebastián, Spain, Departamento de Física de Materiales, Universidad del País Vasco (UPV/EHU), Apartado 1072, 20800 San Sebastián, Spain, and Donostia International Physics Center (DIPC), Paseo Manuel de Lardizabal 4, 20018 San Sebastián, Spain

Prof. J. A. Pomposo

Centro de Física de Materiales (CSIC, UPV/EHU) - Materials Physics Center, Paseo Manuel de Lardizabal 5, 20018 San Sebastián, Spain, Departamento de Física de Materiales, Universidad del País Vasco (UPV/EHU), Apartado 1072, 20800 San Sebastián, Spain, and IKERBASQUE - Basque Foundation for Science, Maria Diaz de Haro 3, 48013 Bilbao, Spain E-mail: josetxo.pomposo@ehu.eus

Endowing unimolecular soft nano-objects with biomimetic functions is attracting significant interest in the emerging field of single chain technology. Inspired by the compartmentalized structure and polymerase activity of metalloenzymes we have designed, synthesized and

\footnotetext{
a $\square$ Supporting information for this article is available at the bottom of the article's abstract page, which can be accessed from the journal's homepage at http://www.macros.wiley-vch.de, or from the author.
} 
characterized copper-containing compact nano-globules endowed with metalloenzyme mimicking characteristics towards controlled synthesis of water-soluble polymers and thermoresponsive hydrogels. When compared to metalloenzymes, artificial nano-objects endowed with metalloenzyme mimicking characteristics offer increased stability against thermal changes and reduced degradability by hydrolytic enzymes.

\section{Introduction}

About one-third of all enzymes known so far are metalloenzymes containing metal ions which are directly bound to the protein or to prosthetic groups (non-amino acid portions required for enzyme function firmly bonded to the protein). ${ }^{1}$ Although enzyme-initiated free radical polymerization of vinyl monomers in the presence of peroxides goes back more than two decades ago, ${ }^{2}$ the use of metalloenzymes per se as catalysts in modern, controlled polymerization processes like atom transfer radical polymerization (ATRP) ${ }^{3}$ or combined ATRP / reversible addition fragmentation chain transfer (RAFT) ${ }^{4}$ polymerization is very recent. These techniques allow fine control over the molecular architecture, molecular weight and polydispersity of synthetic polymers, so controlled enzymatic polymerization broadened significantly their scope.

Metalloenzymatic ATRP (and ATRP/RAFT) was reported by di Lena and co-workers ${ }^{5,6}$ in 2011, involving copper-containing laccase as well as iron-containing catalase and horseradish peroxidase (HRP) enzymes. Independently, Bruns and colleagues ${ }^{7,8}$ discovered that the metalloproteins HRP and (Cys-blocked) hemoglobin were able to polymerize vinyl monomers under reductive conditions, such as those used in activator regenerated by electron transfer (ARGET) ATRP. ${ }^{9}$ Interestingly, HRP was found to be stable during polymerization showing neither unfolding, nor conjugation / oligomerization events. We were intrigued by these discoveries showing polymerase activity* of metalloenzymes under reductive conditions 
and, especially, by the potential feasibility of designing bioinspired synthetic nano-objects having metalloenzyme mimicking properties for the controlled synthesis of water-soluble polymers.

Ultra-small unimolecular soft nano-entities endowed with useful, autonomous and smart functions are the expected, long-term valuable output of single chain technology. ${ }^{10}$ Different soft nano-objects have been synthesized in recent years via single chain technology through chain compaction, such as dynamic, ${ }^{11,12}$ letter-shaped ${ }^{13}$ and compositionally unsymmetrical single rings, ${ }^{14}$ complex multi-ring systems, ${ }^{15}$ single chain nanoparticles, ${ }^{16-19}$ tadpoles, ${ }^{20}$ dumbbells $^{21}$ and hairpins. ${ }^{22}$ In particular, single chain nanoparticles are attractive building blocks for endowing synthetic nano-objects with bioinspired functions, as pioneered by some of us. $^{23-26}$ Single chain nanoparticles are polymeric soft nano-objects consisting of unimolecular chains collapsed to a certain degree by means of intramolecular bonding (i.e., covalent, non-covalent or reversible covalent bonds). ${ }^{16-19}$ The folding/collapse process leading to nanoparticle formation has attracted significant interest as a simplified model of protein folding. Sensing, controlled drug delivery and catalytic applications of single chain nanoparticles have been recently demonstrated. ${ }^{16-19}$

Currently, single chain nanoparticles with two opposite morphologies in solution can be prepared. By performing the folding/collapse process of functional precursors in good solvent, sparse nano-objects are obtained even by employing highly-efficient intra-chain cross-linking techniques (e.g., “click” chemistry) or strong supramolecular interactions. ${ }^{27}$ The sparse morphology is a consequence of the extended conformation of the precursor in good solvent which promotes intra-chain reactions between near neighbor functional groups. Consequently, the actual morphology of single chain nanoparticles synthesized in good solvent is similar to

\footnotetext{
*Along this work, the term polymerase activity refers to the ability of metalloenzymes to catalyze the controlled radical polymerization of water soluble vinyl-type monomers.
} 
the "pearl-necklace" conformation observed in intrinsically disordered proteins (IDPs). ${ }^{23,27}$ On the contrary, globular morphologies in solution have been reported for single chain nanoparticles synthesized by means of folding/collapse of amphiphilic random copolymers in water ${ }^{28}$, due to the formation of individual core-shell nanostructures; a result supported by recent computer simulations. ${ }^{29}$ This morphology is more akin to the globular, native conformation of enzymes showing a hydrophobic core and a hydrophilic shell, as discussed by Terashima et al. $^{28 a}$

Recently, we have reported the synthesis in tetrahydrofuran (THF) of sparse metallofolded SCNPs containing $\mathrm{Cu}(\mathrm{II})$ ions displaying catalytic specificity during the oxidative coupling of mixtures of chemically related terminal acetylene substrates. ${ }^{26 a}$ Using a similar approach, Pd-containing SCNPs have also been reported by Willenbacher et al. with catalytic activity in the Sonogashira coupling. ${ }^{26 \mathrm{~b}}$ The enzyme-mimic character displayed by the Cu(II)containing SCNPs under oxidative conditions motivated us to synthetize their water-soluble globular counterparts, as well as to investigate their catalytic activity under reductive conditions (i.e., conditions of ARGET ATRP). In this communication, we report the design, synthesis and characterization of copper-containing compact nano-globules endowed with metalloenzyme mimicking characteristics towards controlled synthesis of water-soluble polymers and thermoresponsive hydrogels under reductive conditions. To our best knowledge, this is the first report of water-soluble polymers and thermoresponsive hydrogels synthesized by metal-containing single chain globules.

\section{Results and Discussion}

\subsection{Design and synthesis of metalloenzyme mimicking single chain globules}

Scheme 1 illustrates the synthetic approach followed in this work, comprising the preparation of amphiphilic random copolymers which self-assemble in water to unimolecular, globular 
core-shell nanostructures further compacted via single chain technology by means of intrachain copper complexation to give single chain globules with metalloenzyme mimicking polymerase activity.

Hence, a range of representative water-soluble amphiphilic poly(OEGMA-co-AEMA) copolymers composed of hydrophilic oligoethylene glycol methyl ether methacrylate (OEGMA, $M_{\mathrm{n}}=300 \mathrm{Da}$ ) and hydrophobic 2-acetoacetoxy ethyl methacrylate (AEMA) repeat units were first synthesized via RAFT polymerization in the presence of 4-cyanopentanoic acid dithiobenzoate (CPADB) as chain transfer agent. The copolymerization reactions were carried out in 1,4-dioxane to avoid problems arising from the scarce solubility of AEMA in water. Random poly(OEGMA-co-AEMA) copolymers, P1-3, were synthesized showing relatively high values of weight average molecular weight $\left(M_{\mathrm{w}}=47.1-113.6 \mathrm{kDa}\right)$ with narrow molar-mass dispersity $\left(\bigoplus=M_{\mathrm{w}} / M_{\mathrm{n}}=1.07-1.12\right)$ and $12-33$ mol\% of AEMA content, as determined by size-exclusion chromatography (SEC) and ${ }^{1} \mathrm{H}$ NMR spectroscopy (see Supporting Information, SI). This range of AEMA content guarantees both water solubility of the resulting amphiphilic random copolymers and appropriate number of functional groups for further compaction via intra-chain metal complexation.

Next, the synthesis of copper-containing single chain globules, N1-3, from the amphiphilic random copolymers, P1-3, was carried out at r.t. in deionized water, under high dilution conditions ( $1 \mathrm{mg}$ of poly(OEGMA-co-AEMA) / $\mathrm{mL}$ of $\mathrm{H}_{2} \mathrm{O}$ ) by addition of 0.5 equivalent of copper(II) acetate $\left(\mathrm{Cu}(\mathrm{OAc})_{2}\right)$ per equivalent of AEMA (Scheme 1).

\subsection{Characterization of metalloenzyme mimicking single chain globules}

Samples taken periodically from the reaction media were analyzed by SEC. A progressive increase in SEC retention time and, hence, a concomitant reduction in poly(OEGMA-coAEMA) hydrodynamic size was observed as a function of reaction time pointing to a successful compaction via intra-chain $\mathrm{Cu}(\mathrm{II}) /$ (AEMA) $_{2}$ complexation (Fig. 1A). 
Complementary dynamic light scattering measurements performed at r.t. in water showed in all cases a reduction in average hydrodynamic radius $\left(R_{\mathrm{H}}\right)$ upon intra-chain copper complexation (SI, Table S2 and Fig. S1).

Small angle neutron scattering measurements in $\mathrm{D}_{2} \mathrm{O}$ revealed the form factor of compact individual macromolecules ${ }^{30}$ (Fig. 1B), in view of the large exponent of the intensity at intermediate length scales $\left(I(Q) \sim Q^{-3} ; I=\right.$ intensity, $Q=$ wavevector). These results suggest the formation of globular core-shell morphologies with the hydrophobic AEMA units placed in the core. ${ }^{29}$ Direct evidence of Cu(II) / (AEMA) 2 complexation was obtained by FTIR and UV / visible spectroscopies (SI, Fig. S2 and Fig. S3). The amount of copper entrapped within the single chain globules was around 35 mol\% with respect to AEMA units (SI, Table S2 and Fig. S4). Consequently, not all the available AEMA groups were able to form $\mathrm{Cu}(\mathrm{II})$ / (AEMA $)_{2}$ complexes, probably as a consequence of the appearance of progressive conformational restrictions during single chain compaction. The ${ }^{1} \mathrm{H}$ NMR spectra of the copper-containing single chain globules showed significant band broadening, which can be reasonably attributed to the reduced mobility of the protons upon compaction (SI, Fig. S5), as well as to the presence of paramagnetic copper ions. On average, individual single chain globules N1, N2 and N3 contain 33, 16 and $20 \mathrm{Cu}$ atoms per globule, and show $R_{\mathrm{H}}=3.3,2.8$ and $4.3 \mathrm{~nm}$, respectively.

All the above results support the efficient synthesis in water of single chain globules containing $\mathrm{Cu}(\mathrm{II}) /(\mathrm{AEMA})_{2}$ complexes placed in a hydrophobic core, which is stabilized by an external hydrophilic shell.

\subsection{Polymerase activity of metalloenzyme mimicking single chain globules}

The polymerase activity of these bioinspired single chain globules was investigated, as catalysts to produce radicals during controlled polymer synthesis in aqueous media. Table 1 summarizes the main results obtained (fractional conversion $c$, number average molecular 
weight as determined by SEC, $M_{\mathrm{n}, \mathrm{SEC}}$, initiator efficiency, $I_{\mathrm{eff}}$, and molar-mass dispersity, $Ð=$ $\left.M_{\mathrm{w}} / M_{\mathrm{n}}\right)$.

Successful controlled polymerization of OEGMA to linear polyOEGMA was achieved in the presence of ATRP initiator (2-bromopropionitrile), reducing agent $(L-(+)$-ascorbic acid) and RAFT chain transfer agent (2-cyano-2-propyl dithiobenzoate) (Table 1, entries 1-8, and SI) by selecting reaction conditions similar to those reported by di Lena and co-workers ${ }^{5,6}$ but replacing the laccase enzyme ( $4 \mathrm{mg} / \mathrm{mL}$ ) by the copper-containing single chain globules synthesized in this work. Linear pseudo-first kinetic plots were observed in all cases supporting a controlled radical polymerization process $^{3}$ (Fig. 2A and 2B). Even if initiator efficiency was lower than that shown by employing laccase enzyme (i.e., 36-43\%), narrow dispersity values were found for all polyOEGMAs synthesized with the copper-containing single chain globules. The lower initiator efficiency could be the result of a different chemical environment of the $\mathrm{Cu}$ ions in the $\mathrm{Cu}(\mathrm{II}) / \mathrm{AEMA}_{2}$ complexes when compared to the welldefined trinuclear copper cluster of laccase enzyme. As can be seen in Table 1, single chain globules N2 and N3 showing similar copper content but different size (N3 is larger than N2) show similar values of $c, M_{\mathrm{n}, \mathrm{SEC}}$, and $I_{\mathrm{eff}}$ at $50{ }^{\circ} \mathrm{C}$ (entries 4 and 7 , respectively). Single chain globule N1, containing a higher copper content and a size between that of N3 and N2 displays lower $I_{\text {eff }}$ and, consequently, produces polyOEGMA of higher $M_{\mathrm{n}, \mathrm{SEC}}$ and lower $c$ (entry 2 ). For the sake of comparison, polyOEGMA synthesized in the presence of laccase from $T$. versicolor $^{6}$ with identical ATRP initiator, reducing agent and RAFT chain transfer agent showed $c=0.87, M_{\mathrm{n}, \mathrm{SEC}}=36.2 \mathrm{kDa}$ (referred to PS standards), $I_{\mathrm{eff}}=36$ and $Ð=1.8$. No large effect of single chain globule concentration on $c, M_{\mathrm{n}, \mathrm{SEC}}, I_{\mathrm{eff}}$ and $Ð$ was found in the range of 0.8 to $4 \mathrm{mg} / \mathrm{mL}$ (Table 1 , entries 5, 6 and 8 ).

Conversely, in control experiments carried out without initiator or reducing agent no polymerization of OEGMA was observed. In the absence of chain transfer agent, as well as without chain transfer agent and initiator (Table 1, entries 9 and 10), thermoresponsive 
polyOEGMA hydrogels were obtained (Fig. 2C). We hypotesize that the presence of chain transfer agent contributes to eliminate secondary reactions promoting branching at low $c$ and gel formation at high $c$. Interestingly, the temperature at which these polyOEGMA hydrogels were in the collapsed state was around $40{ }^{\circ} \mathrm{C}$, which is in the range of interest for most biomedical applications.

When ARGET ATRP polymerization was carried out from phase-separated systems, such as those involving acrylic acid (AA) (Table 1, entry 11), polymers of very high molecular weight were obtained showing a very broad molecular weight distribution $(\boxplus>2.5)$. On the other hand, poly( $\mathrm{N}$-isopropyl acrylamide-co-acrylic acid), poly(NIPAM-Co-AA), copolymers were successfully synthesized under homogeneous ARGET ATRP conditions with coppercontaining single chain globules as catalyst (Table 1, entry 12). For the sake of comparison, polyNIPAM synthesized from Cys-blocked bovine hemoglobin ${ }^{8}$ under ARGET ATRP conditions showed $c=0.73, M_{\mathrm{n}, \mathrm{SEC}}=200 \mathrm{kDa}$ (referred to PS standards), $I_{\mathrm{eff}}=3.2$ and $Ð=$ 2.2. No problems of stability of the single chain globules were observed at $80^{\circ} \mathrm{C}$ or by the presence of the AA and NIPAM/AA monomers. Concerning the potential reuse of the single chain globules, and similar to the case of metalloenzymes, it was found to depend on the relative solubility of the linear polymer and the globules. Based on the results provided in this communication, evaluation of the potential polymerase activity of other recently reported organometallic nanoparticles would be convenient. ${ }^{26 b, 28 a, 28 d, 28 e}$ Further work is ongoing to investigate the full potential of these water-soluble functional globular nano-objects.

\section{Conclusion}

In summary, by taking inspiration from both the native structure and polymerase activity of laccase metalloenzymes, bioinspired water-borne single chain globules have been designed and constructed via single chain technology through intra-chain copper complexation, allowing controlled synthesis of water-soluble polymers and thermoresponsive hydrogels. 
When compared to metalloenzymes, artificial nano-objects endowed with metalloenzyme mimicking characteristics offer increased stability against thermal changes and reduced degradability by hydrolytic enzymes.

Acknowledgements: We thank Isabel Asenjo-Sanz for her excellent technical support. Financial support from the projects MAT2012-31088 (MINECO) and IT-654-13 (GV) is acknowledged. A. S.-S. thanks the PhD grant support of Basque Government. This work is based on experiments performed at the Swiss spallation neutron source SINQ, Paul Scherrer Institute, Villigen, Switzerland and has been supported by the European Commission under the $7^{\text {th }}$ Framework Programme through the 'Research Infrastructures' action of the 'Capacities' Programme, NMI3-II Grant number 283883.

Keywords: Self-assembly; Nanoparticles; Metal-polymer complexes; RAFT; ATRP.

[1] R. Holm, P. Kennepohl, E. I. Solomon, Chem. Rev. 1996, 96, 2239.

[2] A. Singh, D. L. Kaplan, Adv. Polym. Sci. 2006, 194, 211.

[3] Controlled / Living Radical Polymerization: Progress in ATRP, by K. Matyjaszewski (Ed.); Publisher: American Chemical Society; Washington, D.C., 2009.

[4] Handbook of RAFT Polymerization, by C. Barner-Kowollik (Ed.); Publisher: Wiley-VCH Verlag GmbH \& Co. KGaA, Weinheim, 2008.

[5] Y.-H. Ng, F. di Lena, C. L. L. Chai, Chem. Commun. 2011, 47, 6464.

[6] Y.-H. Ng, F. di Lena, C. L. L. Chai, Polym. Chem. 2011, 2, 589.

[7] S. J. Sigg, F. Seidi, K. Renggli, T. B. Silva, G. Kali, N. Bruns, Macromol. Rapid Commun. 2011, 32, 1710.

[8] T. B. Silva, M. Spulber, M. K. Kocik, F. Seidi, H. Charan, M. Rother, S. J. Sigg, K. Renggli, G. Kali, N. Bruns, Biomacromolecules 2013, 14, 2703. 
[9] J. K. Oh, K. Min, K. Matyjaszewski, Macromolecules 2006, 39, 3161.

[10] a) M. Ouchi, N. Badi, J.-F. Lutz, M. Sawamoto, Nat. Chem. 2011, 3, 917; b) M. Gonzalez-Burgos, A. Latorre-Sanchez, J. A. Pomposo, Chem. Soc. Rev. 2015, DOI: 10.1039/c5cs00209e.

[11] O. Altintas, P. Gerstel, N. Dingenouts, C. Barner-Kowollik, Chem. Commun. 2010, 46, 6291

[12] J. Willenbacher, B. V. K. J. Schmidt, D. Schulze-Suenninghausen, O. Altintas, B. Luy, G. Delaittre, C. Barner-Kowollik, Chem. Commun. 2014, 50, 7056.

[13] B. V. K. J. Schmidt, N. Fechler, J. Falkenhagen, J.-F. Lutz, Nature Chem. 2011, 3, 234.

[14] B. Zhang, H. Zhang, Y. Li, J. N. Hoskins, S. M. Grayson, ACS Macro Lett. 2013, 2, 845.

[15] O. Shishkan, M. Zamfir, M. A. Gauthier, H. G. Börner, J.-F. Lutz, Chem. Commun. 2014, 50, 1570.

[16] C. K. Lyon, A. Prasher, A. M. Hanlon, B. T. Tuten, C. A. Tooley, P. G. Frank, E. B. Berda, Polym. Chem. 2015, 6, 181.

[17] A. Sanchez-Sanchez, J. A. Pomposo, Part. Part. Syst. Charact. 2014, 31, 11.

[18] A. Sanchez-Sanchez, I. Perez-Baena, J. A. Pomposo, Molecules 2013, 18, 3339.

[19] O. Altintas, C. Barner-Kowollik, Macromol. Rapid Commun. 2012, 33, 958.

[20] F. Xu, Z. Fang, D. Yang, Y. Gao, H. Li, D. Chen, ACS Appl. Mater. Interfaces 2014, 6, 6717.

[21] K. Roy, J.-F. Lutz, J. Am. Chem. Soc. 2014, 136, 12888.

[22] J. Lu, N. ten Brummlhuis, M. Weck, Chem. Commun. 2014, 50, 6225.

[23] A. Sanchez-Sanchez, S. Akbari, A. Etxeberria, A. Arbe, U. Gasser, A. J. Moreno, J. Colmenero, J. A. Pomposo, ACS Macro Lett. 2013, 2, 491.

[24] A. Sanchez-Sanchez, S. Akbari, A. J. Moreno, F. Lo Verso, A. Arbe, J. Colmenero, J. A. Pomposo, Macromol. Rapid Commun. 2013, 34, 1681. 
[25] I. Perez-Baena, F. Barroso-Bujans, U. Gasser, A. Arbe, A. J. Moreno, J. Colmenero, J. A. Pomposo, ACS Macro Lett. 2013, 2, 775.

[26] a) A. Sanchez-Sanchez, A. Arbe, J. Colmenero, J. A. Pomposo, ACS Macro Lett. 2014, 3, 439; b) J. Willenbacher, O. Altintas, V. Trouillet, N. Knoefel, M. Monteiro, P. Roesky, C. Barner-Kowollik, Polym. Chem. 2015, DOI: 10.1039/C5PY00389J.

[27] J. A. Pomposo, I. Perez-Baena, F. Lo Verso, A. J. Moreno, A. Arbe, J. Colmenero, ACS Macro Lett. 2014, 3, 767.

[28] a) T. Terashima, T. Mes, T. F. A. De Greef, M. A. J. Gillissen, P. Besenius, A. R. A. Palmans, E. W. Meijer, J. Am. Chem. Soc. 2011, 133, 4742; b) T. Akagi, P. Piyapakorn, M. Akashi, Langmuir 2012, 28, 5249; c) T. Terashima, T. Sugita, K. Fukae, M. Sawamoto, Macromolecules 2014, 47, 589; d) S. Mavila, C. E. Diesendruck, S. Linde, L. Amir, R. Shikler, N.G. Lemcoff, Angew. Chem. Int. Ed. 2013, 52, 5767; e) S. Mavila, I. Rozenberg, N. G. Lemcoff, Chem. Sci. 2014, 5, 4196.

[29] F. Lo Verso, J. A. Pomposo, J. Colmenero, A. J. Moreno, Soft Matter 2015, 11, 1369.

[30] Polymer Physics, by M. Rubinstein and R. H. Colby; Publisher: Oxford University Press; New York, 2003. 


\section{Schemes}

Scheme 1. Synthetic approach towards copper-containing single chain globules from amphiphilic random copolymers composed of hydrophilic oligoethylene glycol methyl ether methacrylate (OEGMA, $\left.M_{\mathrm{n}}=300 \mathrm{Da}\right)$ and hydrophobic 2-acetoacetoxy ethyl methacrylate (AEMA) repeat units.

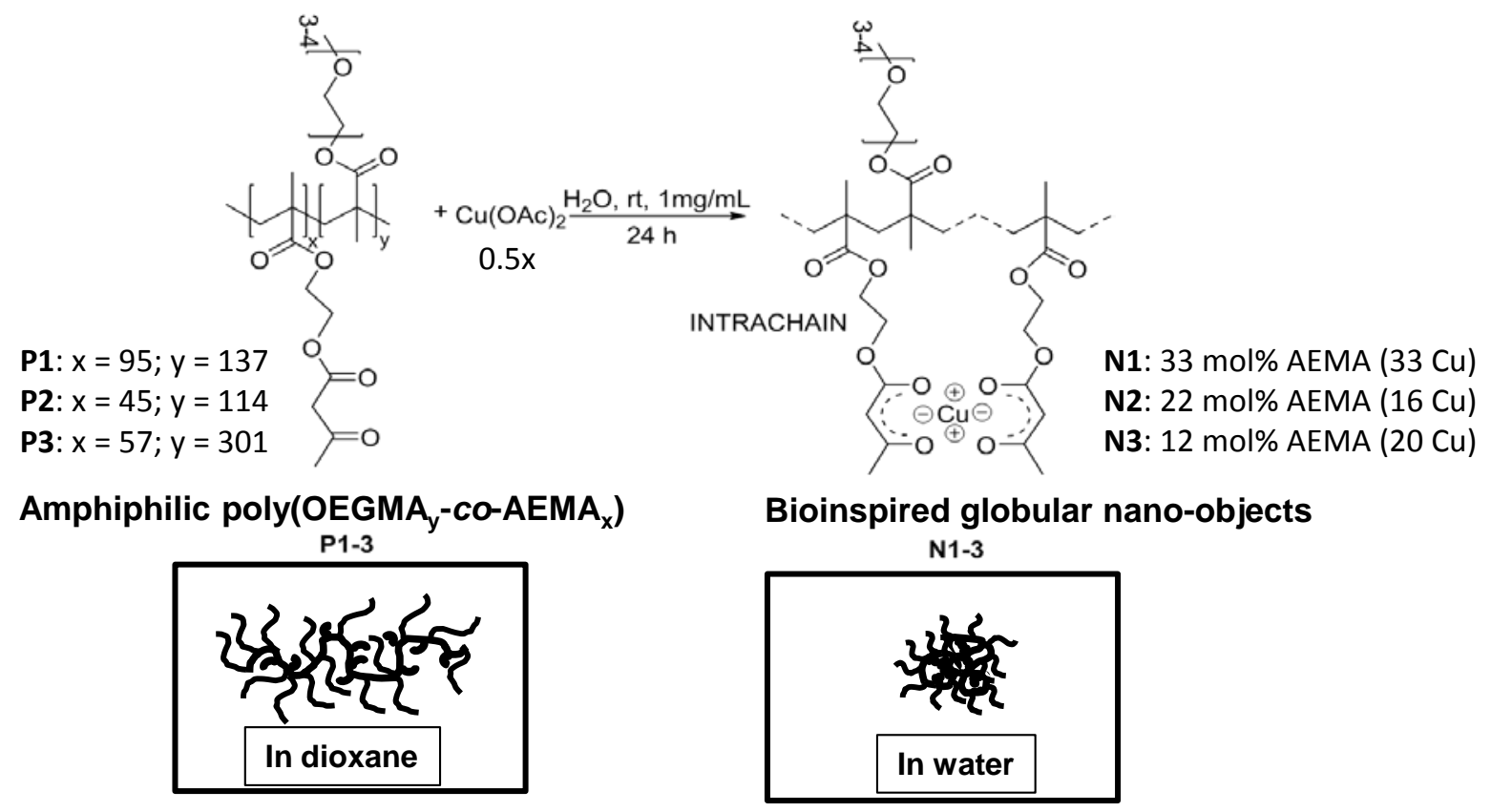




\section{Tables}

Table 1. Summary of the polymerase activity of the metalloenzyme mimicking single chain globules synthesized in this work during polymer synthesis in aqueous media.

\begin{tabular}{|c|c|c|c|c|c|c|c|c|c|}
\hline Entry & Type & $\begin{array}{c}\text { Conc. } \\
(\mathrm{mg} / \mathrm{mL})\end{array}$ & Monomer $^{\mathrm{a}}$ & {$[\mathrm{M}] /[\mathrm{I}] /[\mathrm{A}] /[\mathrm{R}]^{\mathrm{b}}$} & $\begin{array}{c}\mathrm{T} \\
\left({ }^{\circ} \mathrm{C}\right)\end{array}$ & $c$ & $\begin{array}{c}M_{\mathrm{n}, \mathrm{SEC}} \\
(\mathrm{kDa})\end{array}$ & $\begin{array}{c}I_{\mathrm{eff}} \\
(\%)^{\mathrm{c}}\end{array}$ & $\Xi^{d}$ \\
\hline 1 & $\mathbf{N 1}$ & 2 & OEGMA & $50 / 1 / 8 / 0.25$ & 40 & 0.72 & 83.1 & 13 & 1.1 \\
\hline 2 & $\mathbf{N 1}$ & 2 & OEGMA & $50 / 1 / 8 / 0.25$ & 50 & 0.80 & 92.7 & 13 & 1.2 \\
\hline 3 & $\mathbf{N 2}$ & 2 & OEGMA & $50 / 1 / 8 / 0.25$ & 40 & 0.96 & 78.1 & 18 & 1.1 \\
\hline 4 & $\mathbf{N 2}$ & 2 & OEGMA & $50 / 1 / 8 / 0.25$ & 50 & 0.97 & 60.9 & 24 & 1.2 \\
\hline 5 & $\mathbf{N 3}$ & 4 & OEGMA & $50 / 1 / 8 / 0.25$ & 40 & 0.90 & 85.8 & 16 & 1.1 \\
\hline 6 & $\mathbf{N 3}$ & 2 & OEGMA & $50 / 1 / 8 / 0.25$ & 40 & 0.82 & 70.1 & 18 & 1.1 \\
\hline 7 & $\mathbf{N 3}$ & 2 & OEGMA & $50 / 1 / 8 / 0.25$ & 50 & 0.96 & 59.0 & 24 & 1.1 \\
\hline 8 & $\mathbf{N 3}$ & 0.8 & OEGMA & $50 / 1 / 8 / 0.25$ & 40 & 0.97 & 76.5 & 19 & 1.1 \\
\hline 9 & $\mathbf{N 1}$ & 2 & OEGMA & $50 / 1 / 8 / 0$ & 40 & Gel & - & - & - \\
\hline 10 & $\mathbf{N 1}$ & 2 & OEGMA & $50 / 0 / 8 / 0$ & 40 & Gel & - & - & - \\
\hline 11 & $\mathbf{N 2}$ & 2 & AA & $50 / 1 / 8 / 0$ & 80 & 0.72 & 115.5 & 0.02 & 2.8 \\
\hline 12 & $\mathbf{N 2}$ & 2 & NIPAM/AA & $50 / 1 / 8 / 0$ & 50 & 0.63 & 50.7 & 6.4 & 1.8 \\
\hline
\end{tabular}

${ }^{\mathrm{a}}$ OEGMA = Oligoethylene glycol methyl ether methacrylate $\left(M_{\mathrm{n}}=300 \mathrm{Da}\right), \mathrm{AA}=$ Acrylic acid, NIPAM $=N$ Isopropyl acrylamide. ${ }^{\mathrm{b}} \mathrm{M}=$ Monomer, $\mathrm{I}=$ Initiator, $\mathrm{A}=$ Reducing agent, $\mathrm{R}=$ Chain transfer agent. ${ }^{\mathrm{c}} I_{\text {eff }}=$ $\left.\left[\left([\mathrm{M}]_{0} /[\mathrm{I}]_{0}\right) \times c \times M_{\text {monomer }}\right) / M_{\mathrm{n}, \mathrm{SEC}}\right] \times 100 .{ }^{\mathrm{d}} Ð=M_{\mathrm{w}} / M_{\mathrm{n}}$. 


\section{Figures}
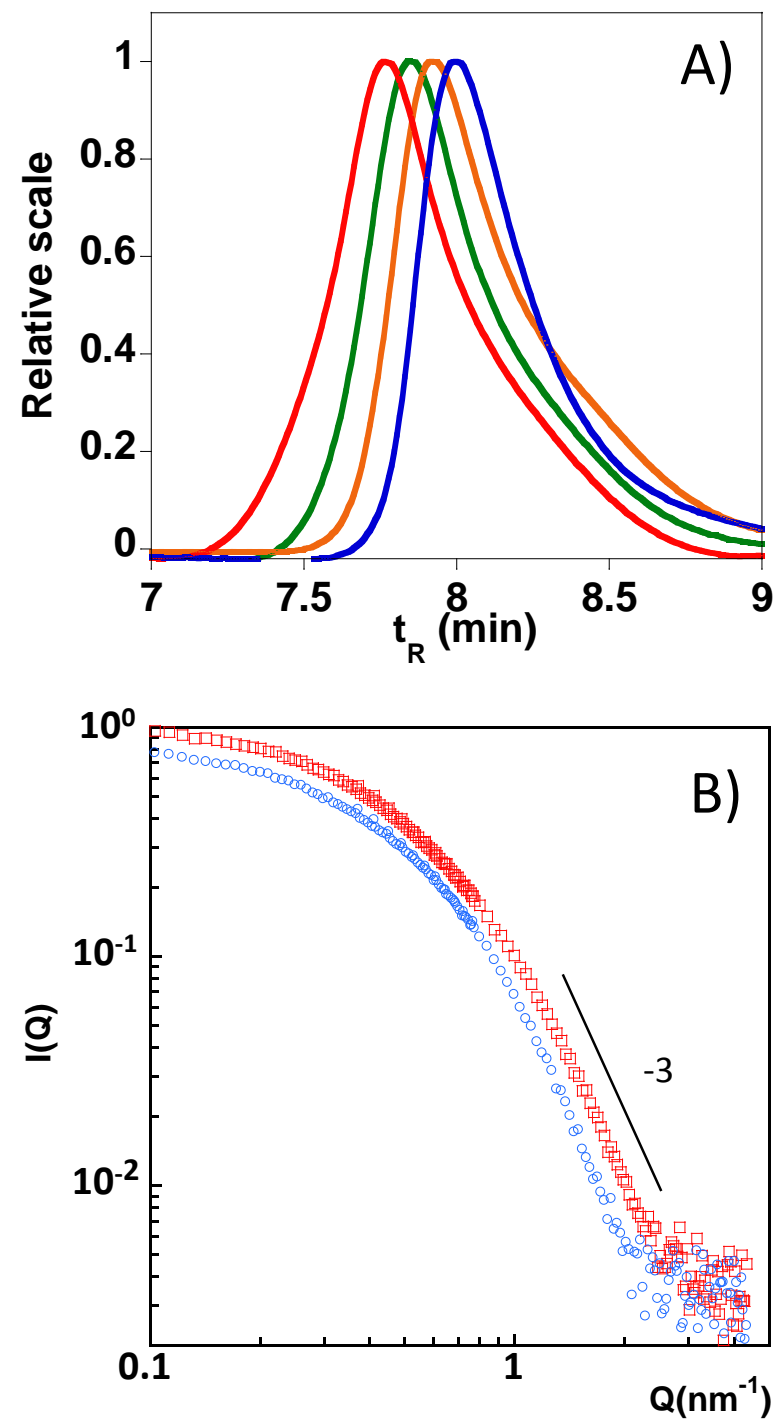

Fig. 1. A) Size-exclusion chromatography traces showing the typical progressive reduction in hydrodynamic size of amphiphilic random poly(OEGMA-co-AEMA) copolymers (P2) upon intra-chain copper complexation in water. Reaction time: 0 h (red curve), 6 h (green curve), $12 \mathrm{~h}$ (orange curve), and $24 \mathrm{~h}$ (blue curve). B) Small angle neutron scattering results in $\mathrm{D}_{2} \mathrm{O}$ support the globular morphology of neat (P2, red symbols) and copper-containing (N2, blue symbols) amphiphilic poly(OEGMA-co-AEMA) copolymers. 

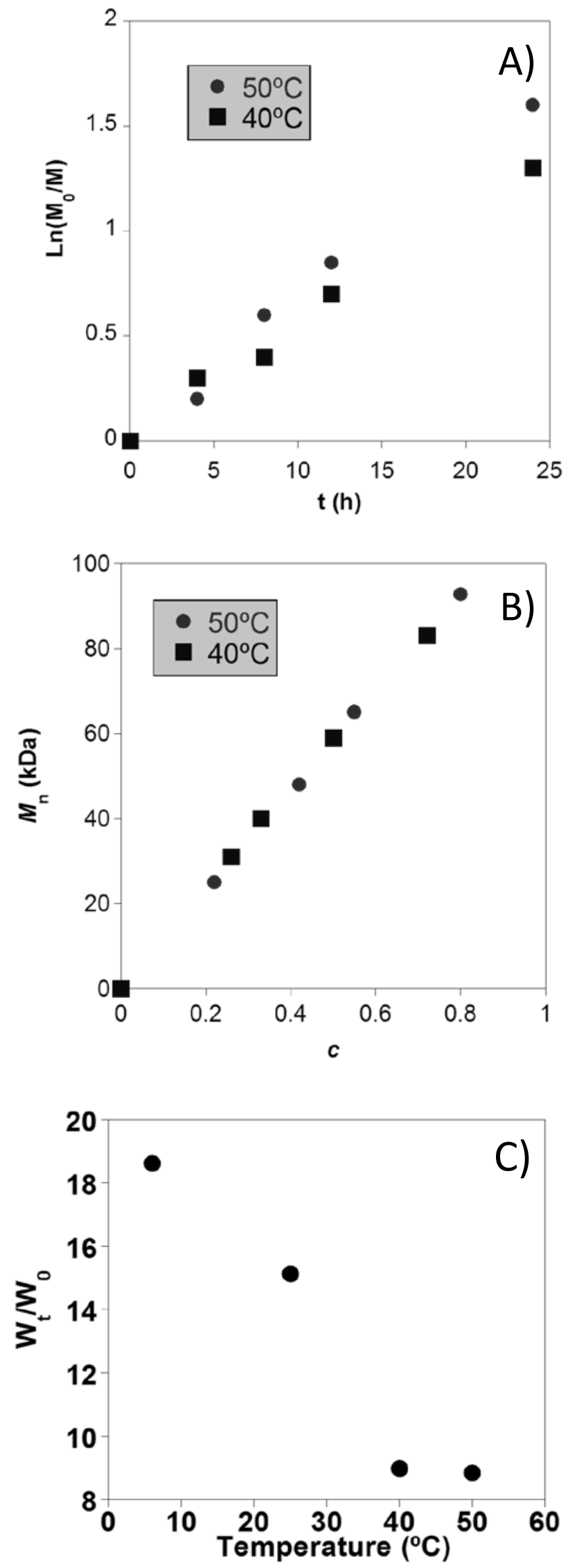
Fig. 2. A) First order kinetic plot for polymerization of OEGMA in the presence of coppercontaining single chain globules for two different temperatures (entries 1 and 2, Table 1). B) Evolution of number-average molecular weight $\left(M_{n}\right)$, as determined by size-exclusion chromatography, with fractional monomer conversion (c) for polyOEGMA synthesized in the presence of copper-containing single chain globules at two different temperatures (entries 1 and 2, Table 1). C) Thermoresponsive properties of polyOEGMA hydrogels synthesized in the presence of copper-containing single chain globules (entry 9, Table 1 ). $W_{\mathrm{t}}$ and $W_{0}$ are the weights of the swollen hydrogel and the dried sample, respectively. At $40{ }^{\circ} \mathrm{C}$ the hydrogel was in the collapsed state. 
Metalloenzyme mimicking characteristics of bioinspired water-borne unimolecular globules constructed via single chain technology allow controlled synthesis of water-soluble polymers and thermoresponsive hydrogels.

Ana Sanchez-Sanchez, Arantxa Arbe, Joachim Kohlbrecher, Juan Colmenero, José A. Pomposo*

Efficient Synthesis of Single Chain Globules Mimicking the Morphology and Polymerase Activity of Metalloenzymes

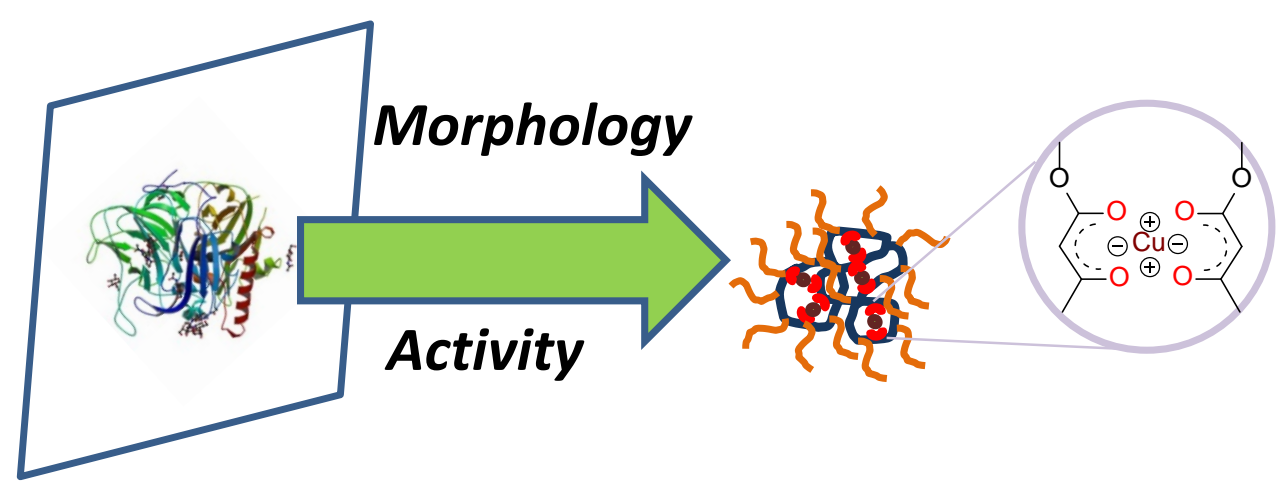

